\title{
A Case of Secondary Aortoduodenal Fistula Diagnosed by Esophagogastroduodenoscopy
}

\author{
Kyosuke Goda ${ }^{1}$, Masanori Kawaguchi ${ }^{2}$ and Akiko Shiotani ${ }^{1}$ \\ ${ }^{1}$ Division of Gastroenterology, Department of Internal Medicine, Kawasaki Medical School, Okayama, \\ Japan \\ ${ }^{2}$ Department of Gastroenterology, Saiseikai Wakayama Hospital, Japan
}

*Corresponding author: Kyosuke Goda, M.D., Division of Gastroenterology, Department of Internal Medicine, Kawasaki Medical School, 577 Matsushima, Kurashiki City, Okayama Prefecture 701-0192, Japan, Tel: 81-86-462-1111, Fax: 81-86464-1195

\begin{abstract}
A 64-year-old man underwent vascular graft surgery for an abdominal aortic aneurysm one year previously. Using esophagogastroduodenoscopy (EGD), he was definitively diagnosed as having an aortoenteric fistula (AEF) and underwent an urgent surgical procedure for treatment. Generally, the prognosis in cases of AEF after vascular graft operation remains poor, and the survival rate in cases of $A E F$ that require surgery is low. We report this case of $A E F$ because EGD played a significant role in its early detection and definitive diagnosis, which resulted in the patient's survival. EGD is not a common procedure for patients with severe symptoms. Therefore, it is necessary to accumulate and analyze data on more cases concerning the criteria for adoption of EGD, which will allow for a more accurate and timely AEF diagnosis in the future.
\end{abstract}

\section{Keywords}

Aortoduodenal fistula, Esophagogastroduodenoscopy, Vascular graft

\section{Introduction}

Even with current advancements in vascular surgery, an aortoenteric fistula (AEF) in the abdominal aortoiliac region after revascularization is very difficult to treat. $A E F$ is classified as a primary fistula, in which the aorta directly penetrates the gastrointestinal tract because of an aneurysm or infection, or a secondary fistula, which develops after graft implantation [1]. The incidence of secondary AEFs is 0.36 to $1.6 \%$ in postoperative surgical cases of abdominal aneurysm [2]. The duration between the first surgery and onset of secondary AEF greatly varies, from one month to 21 years, with an average of approximately 3 to 7 years $[3,4]$. Secondary AEFs are difficult to treat and are significantly fatal; 30 to $70 \%$ of patients die as a result of sepsis or hemorrhagic shock due to gastrointestinal hemorrhage [5].

We encountered a case of secondary aortoduodenal fistula that developed one year after a surgical operation on an abdominal aortic aneurysm and succeeded in detecting and making an early definitive diagnosis of this fistula using esophagogastroduodenoscopy (EGD), ultimately leading to patient survival. Here, we report this case and discuss the case on the basis of other reports.

\section{Case Report}

\section{Chief complaint}

Persistent lower abdominal pain, lower back pain, and anorexia.

\section{Past medical history}

A 64-year-old male had hypertension, dyslipidemia, arteriosclerosis obliterans (treated via a left femoralsupergenual popliteal bypass surgery in 2006 and thrombectomy and another bypass surgery thereafter), abdominal aortic aneurysm and aneurysm of the common iliac arteries (treated via a vascular graft surgery in 2009), incisional hernia (treated via surgical repair in 2009), and transient ischemic attack (in 2009).

Citation: Goda K, Kawaguchi M, Shiotani A (2019) A Case of Secondary Aortoduodenal Fistula Diagnosed by Esophagogastroduodenoscopy. Clin Med Rev Case Rep 5:250. doi.org/10.23937/23783656/1410250

Accepted: January 01, 2019: Published: January 03, 2019

Copyright: (c) 2019 Goda K, et al. This is an open-access article distributed under the terms of the Creative Commons Attribution License, which permits unrestricted use, distribution, and reproduction in any medium, provided the original author and source are credited. 


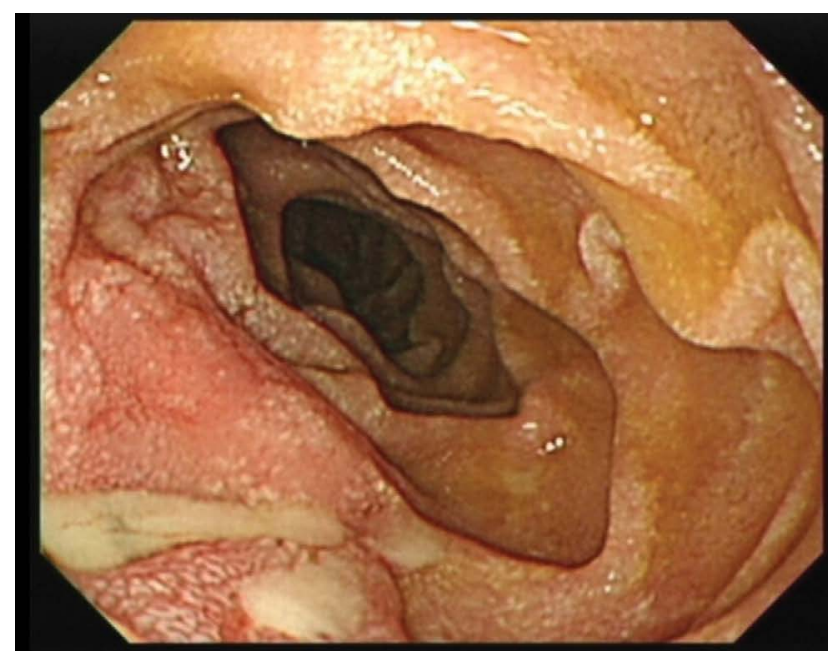

Figure 1: Ulceration with white color on the surface was found in the third portion of the duodenum. Purulent exudate was found in the lesion.

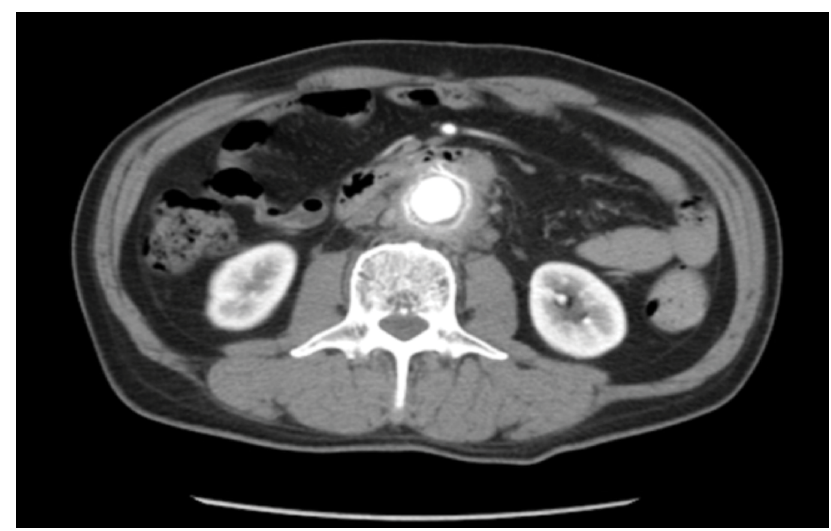

Figure 2: Increased adipose concentration was found around the abdominal aorta without clear boundaries. Some inflammatory response or the onset of retroperitoneal fibrosis was suspected.

\section{History of present illness}

This patient had undergone blood vessel replacement surgery as treatment for an abdominal aortic aneurysm and was undergoing postoperative follow-up as an outpatient. The patient presented to the hospital with chief complaints of persistent lower abdominal pain, lower back pain, and anorexia. The patient did not have hematemesis, nor did he have melena.

\section{Condition at first visit}

This patient was $162 \mathrm{~cm}$ tall, weighed $59 \mathrm{~kg}$, and had a body temperature of $36.7{ }^{\circ} \mathrm{C}$, clear consciousness, normal heart sounds, no hepatosplenomegaly, no clear tenderness in the abdomen, no rebound tenderness, and no costovertebral angle (CVA) tenderness. Intestinal peristaltic sounds were audible.

\section{Blood count and biochemical findings}

Hemoglobin, $12.8 \mathrm{~g} / \mathrm{dl}$, White blood cells (WBC) count, $8,000 / \mathrm{mm}^{3}$ and C-reactive protein (CRP) level, $3.9 \mathrm{mg} / \mathrm{dL}$, signifying mildly increased inflammatory response.

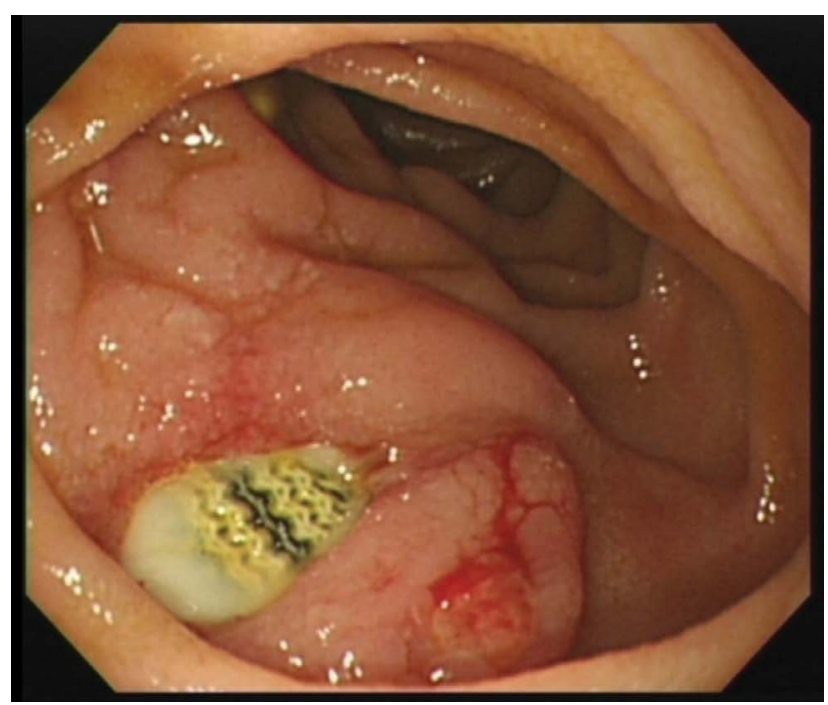

Figure 3: The graft was exposed on the duodenal side and pierced there in the ulcer area that had been found in the previous EGD inspection.

\section{Blood culture}

Negative.

\section{EGD (Figure 1)}

Ulceration with a whitish surface was found in the third portion of the duodenum. A purulent exudate was found in the lesion.

\section{Abdominal computed tomography (CT) findings (Figure 2)}

Increased adipose concentration was found around the abdominal aorta without clear boundaries. An inflammatory response or onset of retroperitoneal fibrosis was suspected.

\section{Progress after hospitalization}

The patient was diagnosed as having a duodenal ulcer and was treated through complete fasting with the administration of a proton pump inhibitor (PPI) and an antibiotic, with observation of his progress. His temperature did not increase; however, his abdominal symptoms did not improve. Blood tests performed one week after hospital admission showed an increased inflammatory response, with WBC levels of 18,500/ $\mathrm{mm}^{3}$ and CRP levels of $30.8 \mathrm{mg} / \mathrm{dL}$. Therefore, an aortoduodenal fistula was suspected, and the EGD was repeated in the presence of surgeons. It was then found that the graft was exposed on the duodenal side and torn in the ulcerative area that was found on the previous EGD (Figure 3). Therefore, the patient was definitively diagnosed as having a secondary aortoduodenal fistula. After consultation with cardiovascular surgeons and general surgeons, the patient was treated via an urgent surgery. After the fistula was confirmed, the third portion of the duodenum was resected. The greater omentum was fixed there such that it covered the graft.

The patient received antibiotics and was subjected 
to complete fasting under follow-up observation. He had his first meal eight days postoperatively. Twentythree days postoperatively, he was under follow-up observation using computed tomography (CT) and EGD. He was discharged on the $40^{\text {th }}$ postoperative day because his condition had improved and was stable.

\section{Discussion}

Regarding the mechanism of fistula formation in secondary AEF cases, there are two theories: Infection and physical stimulation. The infection theory suggests that if there is an infection in the anastomosis site of a blood vessel prosthesis, the infected anastomosis would open and enlarge, a pseudo-aneurysm would form, and the aneurysm would cause erosion and inflammation of the enteric tube, which would result in a fistula [5] However, the physical stimulation theory is that the anastomosis site of a blood vessel prosthesis would adhere to the enteric tube, and aortic beats would cause erosion in the enteric tube, which would result in the aorta penetrating the tube [6]. Approximately $80 \%$ of secondary AEFs are formed in the third portion of the duodenum [7]. This disease presents with a triad of gastrointestinal hemorrhage, abdominal pain, and abdominal pulsating mass; however, only $25 \%$ of reported cases have all three signs [2].

The present case was a case of secondary AEF that developed approximately one year after a blood vesselreplacement surgery. When the patient first visited our department, he only complained of abdominal pain and anorexia, and the AEF triad was not found. He was found to have an increased WBC and inflammatory response, and a CT scan indicated a suspected inflammatory response around the graft; therefore, we assumed that infection of the graft might have caused the onset of secondary AEF. However, blood culture was negative, and no clear pseudo-aneurysms were found during the operation. Therefore, it is possible that physical stimulation of aortic beats might have caused fistula formation.

In principle, treatment for AEF consists of hemorrhage control, infection control (closure of the gastrointestinal tract, drainage, debridement, etc.), removal of infected blood vessel prostheses, and revascularization [2]. Patients with minor infections are very likely to survive if the AEF is treated locally, with few perioperative complications [8]. The question of what surgical technique is the best for such cases should be considered on a case-by-case basis, according to systemic conditions and the onset mechanism.

In the present case, the graft was cleaned and disinfected but not removed, and no revascularization was performed. The postoperative course was good. Considering the facts that the patient had no hemorrhagic shock, was in good systemic condition, was definitively diagnosed preoperatively, and had no pseudo-aneurysms, the graft seemed to have only minor infections. Under these circumstances, the selected surgical approach seemed appropriate.

It was reported that approximately $80 \%$ of the secondary AEF cases with herald bleeding had a fatal massive hemorrhage within 24 hours to one week after the herald bleeding $[9,10]$. Secondary AEF is a rare disease; moreover, its triad is not found in many cases. Therefore, it is relatively difficult to diagnose, and it has been reported that in some cases, a late diagnosis resulted in patient death [11]. Accordingly, standards for the diagnosis of this disease and predictive factors for early detection should be considered and clarified.

For early detection of this disease, a contrast CT scan is recommended $[12,13]$. However, contrast CT findings could not form the basis of a definitive diagnosis or urgent surgery in the present case because the patient had no herald bleeding and had relatively stable vital signs. Because the abdominal pain did not improve, and the inflammatory response deteriorated, we suspected secondary AEF and repeated the EGD, which led to a definitive diagnosis of the disease, resulting in surgery on the patient. In view of the above, if a contrast CT scan is not helpful in making a definitive diagnosis in cases wherein a past history is suggestive of secondary AEF without herald bleeding, an EGD may be of great help. Nevertheless, in cases of AEF with pseudo-aneurysm formation, it is possible that EGD may worsen the hemorrhage in a fistula. Therefore, in a situation in which EGD is to be performed on a patient with suspected AEF, it is desirable to cooperate with and consult vascular surgeons. Moreover, EGD is not typically performed on patients with severe symptoms. Therefore, it is necessary to accumulate and analyze data on more cases concerning the adaptation criteria for EGD, which will allow for more accurate and timely diagnoses of $A E F$ in the future.

In conclusion, the present case reports the survival of a patient with a secondary aortoduodenal fistula that developed one year after surgery on an abdominal aortic aneurysm. The disease was detected early and diagnosed definitively using EGD, which led to the patient's survival. The possibility of encountering this disease should be kept in mind during daily clinical practice.

There are no conflicts of interest to declare.

\section{References}

1. Sugawara $Y$, Mohri N, Nagae T (2005) A case of abdominal aortic aneurysm ruptured into duodenum. The journal of the Japan Surgical Association 66: 1590-1594.

2. Busuttil SJ, Goldstone J (2001) Diagnosis and management of aortoenteric fistulas. Semin Vasc Surg 14: 302-311.

3. Bergqvist $D$, Bjorkman $\mathrm{H}$, Bolin $\mathrm{T}$, Dalman $\mathrm{P}$, Elfström $\mathrm{J}$, et al. (1996) Secondary aortoenteric fistulae-changes from 1973 to 1993. Eur J Vasc Endovasc Surg 11: 425-428. 
4. Champion MC, Sullivan SN, Coles JC, Goldbach M, Watson WC (1982) Aortoenteric fistula. Incidence, presentation recognition, and management. Ann Surg 195: 314-317.

5. Matsuura K, Takahara Y, Sudo Y, Ishida K, Nakajima N (2002) A case of fistula of the right common iliac aneurysm to the appendix. Ann Vasc Surg 16: 768-770.

6. Bunt TJ (1983) Synthetic vascular graft infections. Graftentericerosions and graft-enteric fistulas. Surgery 94: 1-9.

7. Akimoto $K$, Nagai A, Sugiyama S, Hamanaka H, Ueyama $T$ (1987) Survival of a case of aortoduodenal fistula developing after replacement surgery with Y-shaped artificial graft. Operation 41: 1851-1854.

8. Oda Y, Kawahara S, Ogawa S (1993) Two autopsy cases of abdominal atherosclerotic aneurysm with rupture into the duodenum. Pathology and Clinical Medicine 11: 353-359.
9. Thomas WEG, Baird RN (1986) Secondary aorto-enteric fistulae: Towards a more conservative approach. Br J Surg 73: $875-878$.

10. Steffes BC, O Leary JP (1980) Primary aortoduodenal fistula: A case report and review of the literature. Am Surg 46: 121-129.

11. Kawahara K, Okada M, Hayashi T, Fujita Y, Sasaki M, et al. (2001) An autopsy of a primary aortoduodenal fistula case complicated by both gastric carcinoma and acute gastric mucosal lesions. Rinsho Byori 49: 77-81.

12. Nakao M, Maeda A, Kinugawa $Y$ (2010) A case of surgical operation on primary aorto-enteric fistula found due to massive bloody stool. Jpn J Vasc Surg 19: 505-508.

13. Ito S, Kubota K, Kogure H (2001) A case of secondary aorto-duodenal fistula for which abdominal CT scanning was useful. Jpn J Gastroenterol Surg 34: 586-589. 